\title{
Efficient Image Updates Using Light Fields*
}

\author{
Biswarup Choudhury \\ Computer Science \& Engg. Dept. \\ IIT Bombay \\ Mumbai, India 400076
}

\author{
Aviral Pandey \\ Institute of Technology \\ Banaras Hindu University \\ Varanasi, India 221005 \\ aviral.pandey@cse05.itbhu. org
}

\author{
Sharat Chandran \\ Computer Science \& Engg. Dept. \\ IIT Bombay \\ Mumbai, India 400076 \\ sharatecse.itit.ac.in
}
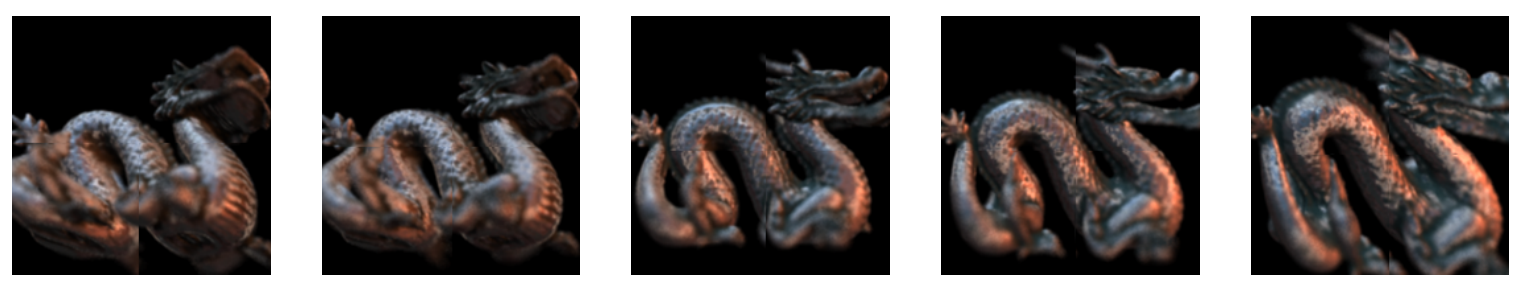

\begin{abstract}
The light field rendering method is an interesting variation on achieving realism. Once authentic imagery has been acquired using a camera gantry, or a handheld camera, detailed novel views can be synthetically generated from various viewpoints.

One common application of this technique is when a user "walks" through a virtual world. In this situation, only a subset of the previously stored light field is required, and considerable computation burden is encountered in processing the input light field to obtain this subset. In this paper, we show that appropriate portions of the light field can be cached at select "nodal points" that depend on the camera walk. Once spartanly and quickly cached, scenes can be rendered from any point on the walk efficiently.
\end{abstract}

\section{Introduction}

The traditional approach for "flying" through scenes is by repeated rendering of a three-dimensional geometric model. One well known problem with "geometry-based" modeling is that it is very difficult to achieve photo-realism due to the complex geometry and lighting effects present in nature. A relatively newer approach is Image-Based Rendering (IBR [2], [3]) which uses a confluence of methods from computer graphics and vision. The IBR approach is to generate novel views from virtual camera locations from pre-acquired imagery. Synthetic realism is achieved, so to speak, using real cameras.

Light Field Rendering (LFR) [5] (or Lumigraphs [4]) is an example of IBR. The approach here is to store samples

\footnotetext{
*An earlier version of this paper appeared in ICVGIP 2004 [8]
}

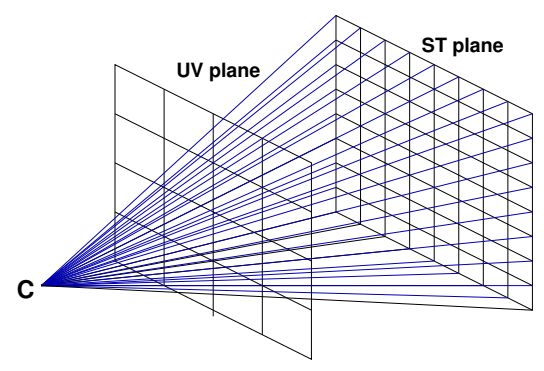

Figure 1. Two-Plane Parametrization

of the plenoptic function[1] which describes the directional radiance distribution for every point in space. The subset of this function in an occlusion-free space outside the scene can be represented in the form of a four-dimensional function. The parameterization scheme is shown in Figure 1. Every viewing ray, computed using a ray-shooting technique, from the novel camera location $C$ passing through the scene is characterized by a pair of points $(s, t)$ and $(u, v)$ on two planes. By accessing the previously acquired radiance associated with this four tuple, we are able to generate the view from $C$. In order to view a scene from any point in surrounding space, six light slabs are combined so that the six viewpoint planes cover some box surrounding the scene.

\subsection{Statement of the Problem}

The beauty of LFR lies in re-sampling and combining the pre-acquired imagery. In a typical walk-through situation, a person is expected to walk along a trajectory in three space and "suitably" sample the light field. The problem we pose in this paper is "Given the light field on disk, and a walk using a camera, how fast can the images seen by the camera be updated?" 
For best results in light field based IBR, we expect that the size of the light field data-structure drastically increases with the increase in the resolution and the density of acquired images. As mentioned above, ray-shooting is performed as an intermediate step of the rendering procedure, a reportedly computationally intensive operation [9].

\subsection{Contributions}

For interactive rendering of the scene, one needs to store the complete light field in volatile memory, whereas only a subset of this is needed for a specific camera walk. Prior methods do not effectively address this issue. In this paper, we show how caching the light field suitable for the camera walk, dramatically reduces the computational burden, this is seen in Figure 10. Specifically,

- We compute the optimal location of a sparse set of "nodal points." The lightweight "light field" stored at these nodes is enough to render the scene from any of the infinite points - termed query points - on the camera path.

- The method in [9] uses homography to reduce the ray shooting computational burden in producing the image from one query point; multiple query points are treated afresh since no notion of nodal points was required therein. We use an alternative Taylor series method for reducing the computational burden of ray shooting queries; this is particularly applicable when the center of projection of the camera moves along a plane parallel to the UV and ST planes.

- The correctness of our scheme is shown using a mathematical characterization of the geometry of the light field. Experimental results validate our scheme.

The rest of this paper is organized as follows. Section 2 develops the mathematical framework for the problem. In Section 3, we give details of our approach and present our algorithm. Sample results and analyses are given in Section 4. We end with some concluding remarks in the last section.

\section{Our Approach}

As in the original work [5], the field of view of the query camera is expected to be identical to the cameras that generated the light field. Likewise, sheared perspective projection [5] handles the problem of aligning the plane of projection with the light-slab. Coming to the camera walk, in this section we provide the mathematical basis for the location and spacing of nodal points.

For brevity, we initially restrict the center of projection of the camera to move along a plane parallel to the UV and the ST plane. We later relax this condition and show that, the mathematical framework still holds. For motivation, consider a setup similar to the two slab setup where planes, UV and ST are replaced by lines $\mathrm{U}$ and S. We call this as the two-line setup. The query points lie on line $\mathrm{C}$, which in turn replaces the camera plane. We provide the complete mathematical framework with respect to this setup.

\subsection{Fixed Direction}

The algorithms in this section tell us where to place nodal points for a specific query point $q$ assuming a fixed direction determined by some point $s$. This condition is relaxed later.

Denote $\Delta l$ to be the constant distance $d\left[G_{i}, G_{i+1}\right]$ between two consecutive grid points on the $U V$ plane, i.e., the distance between the acquired camera locations.

\subsubsection{Fixed Direction Algorithm}

Given $q$, Algorithm 2.1.1 computes nodal points $N_{1}$ and $N_{2}$ (Figure 2). The radiance $L[q]$, in the direction of $s$, is presumably cached at points $N_{1}$ and $N_{2}$. We need to make use of this cache. Denote $\operatorname{assoc}(\mathrm{p})$, where $p$ is a point

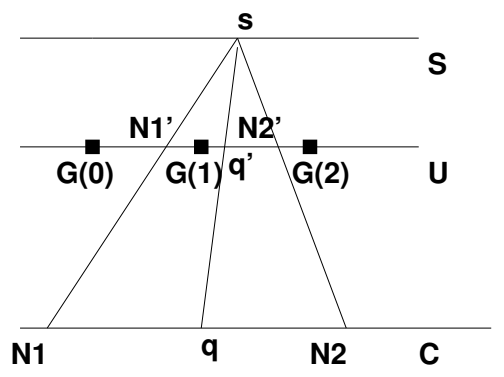

Figure 2. $N_{1}$ and $N_{2}$, the nodal points for q are marked such that $d\left[q^{\prime} N_{1}^{\prime}\right]=d\left[q^{\prime} N_{2}^{\prime}\right]=\frac{\Delta l}{2}$.

on $\mathrm{C}$, to be the closest grid vertex $G$ (on $\mathrm{U}$ ) to the ray $\overline{p s}$. Suppose assoc (q) is $G_{i}$.

\begin{tabular}{|l|}
\hline Algorithm 2.1.1: Fixed-Direction $(q, s)$ \\
\hline \hline Shoot a ray from $q$ to $s$ to obtain $q^{\prime}$ on U. \\
Mark points $N_{1}{ }^{\prime}$ and $N_{2}{ }^{\prime}$ on $\mathrm{U}$ at a distance $d=\frac{\Delta l}{2}$ \\
apart on either side of $q^{\prime}$. This determines the nodal \\
points $N_{1}$ and $N_{2}$ on C. \\
if a s $\circ C\left(N_{1}\right)$ is $G_{i}$ then \\
$\quad L[q]=L\left[N_{1}\right]$ \\
else \\
$\quad L[q]=L\left[N_{2}\right]$ \\
end if \\
\hline
\end{tabular}

In the two-dimensional case, given $q$, our algorithm computes four nodal points $N_{1}, N_{2}, N_{3}$ and $N_{4}$. Shoot the ray from $q$ to $s$ for a given $s$ to obtain $q^{\prime}$ on UV. Now, mark four 
points $\left(q^{\prime} \cdot u \pm \frac{\Delta l}{2}, q^{\prime} \cdot v \pm \frac{\Delta l}{2}, z_{u v}\right)$, where $q^{\prime} \cdot u$ and $q^{\prime} \cdot v$ represent the component of $q^{\prime}$ along $u$ and $v$ respectively. These four points correspond to four nodal points on the camera COP (center of projection) plane. We use assoc of these nodal points to determine $L[q]$.

\subsubsection{Comments}

Notice that if the distance $d$ in Algorithm 2.1.1 is more than $\frac{\Delta l}{2}$, as in Figure 3, an incorrect value of $L[q]$ is computed. When $d$ is as specified in Algorithm 2.1.1, it is easy to observe that either assoc $\left(N_{1}\right)=G_{1}$ or assoc $\left(N_{2}\right)$ $=G_{1}$; it cannot be the case that assoc $\left(N_{1}\right)=G_{0}$ and assoc $\left(N_{2}\right)=G_{2}$. A choice less than $\frac{\Delta l}{2}$ might be suitable to maintain correctness, but will increase the number of nodal points, and hence decrease our efficiency.

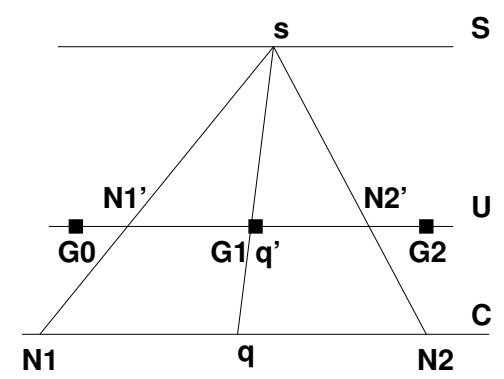

Figure 3. $\operatorname{assoc}\left(N_{1}\right)$ is $G_{0}$ and assoc $\left(N_{2}\right)$ is $G_{2}$.

\subsection{Changing the view direction}

The results in this section assert that the nodal points may be chosen by arbitrarily picking any direction and applying Algorithm 2.1.1. That is, the selection of nodal points is independent of the direction.

Lemma 2.2.1 The set of nodal points $\{N 1, N 2\}$ for a given point $s$ on $S$, serves as the set of nodal points for all s.

Proof: For any query point $q$, consider two directions corresponding to $s 1$ and $s 2$. Let $N_{1}$ be any nodal point determined by Algorithm 2.1.1 for $q$ and $s 1$. The lemma asserts that nodal point $N_{1}$ serves as a nodal point for $q$ and $s 2$. Figure 4 illustrates the idea.

The basic idea is to prove that $N_{1}^{\prime} q^{\prime}$ is equal to $N_{1}^{\prime \prime} q^{\prime \prime}$. Applying similar triangles on $\Delta\left[N_{1}, S_{1}, q\right]$ and $\Delta\left[N_{1}, S_{2}, q\right]$, this can easily be proved.

Next, we consider the lemma for the two-dimensional case:

Lemma 2.2.2 Given a query point, nodal points may be decided using any point $(s, t)$ provided the camera planes are parallel.
Proof: As in Figure 5, let $N_{1}, N_{2}, N_{3}, N_{4}$ be the nodal points for query point $q$ as determined by Section 2.1.1. Let $S_{a}=\left(s_{1}, t_{1}, z_{s}\right)$ be the intersection point of the query ray from q on the ST plane, and let $X_{a}=\left(x_{1}, y_{1}, z_{u}\right)$ be the intersection point of that ray on the UV plane. Similarly, define $S_{b}$ and $X_{b}$. The proof uses the relationships (equations 1 and 2) below, to prove equation 3 .

$$
\begin{gathered}
\overline{S_{b} N 1}=\overline{S_{a} N_{1}}+\overline{\left(S_{a}-S_{b}\right)} \\
\overline{S_{b} q}=\overline{S_{a} q}+\overline{\left(S_{a}-S_{b}\right)} \\
\overline{S_{b} N 1}=k\left(\overline{S_{b} X_{b}}+\left(-\frac{\Delta l}{2}, \frac{\Delta l}{2}, 0\right)\right)
\end{gathered}
$$

\subsection{The Power of Nodal Points.}

Once nodal points are selected, there are a range of query points for which these nodal points are valid, as stated below.

Lemma 2.3.1 The nodal points $N_{1}, N_{2}$ of a query point $q_{1}$ are sufficient for determining the radiance of any query point in the interval $\left[N_{1}, N_{2}\right]$.

Proof: Consider any point $q_{2}$, between $N_{1}$ and $N_{2}$, and let the nodal points as determined by Algorithm 2.1.1, be $N_{3}$ and $N_{4}$. The lemma asserts that, for $q_{2}$, the radiance values stored at $N_{1}$ and $N_{2}$ are sufficient.

Without loss of generality, assume $q_{2}$ to be nearer to $N_{2}$ than $N_{1}$. We observe that either $d\left[N_{1}{ }^{\prime}, \operatorname{assoc}\left(N_{3}\right)\right]<\frac{\Delta l}{2}$ or $d\left[N_{2}^{\prime}, \operatorname{assoc}\left(N_{3}\right)\right]<\frac{\Delta l}{2}$.

- Case 1: $d\left[N_{1}{ }^{\prime}, \operatorname{assoc}\left(N_{3}\right)\right]<\frac{\Delta l}{2}$ (shown in Figure $6(\mathrm{a})) \Rightarrow \operatorname{assoc}\left(\mathrm{N}_{3}\right)=\operatorname{assoc}\left(\mathrm{N}_{1}\right)$. So $\operatorname{assoc}\left(\mathrm{N}_{2}\right)=\operatorname{assoc}\left(\mathrm{N}_{4}\right)$, i.e., $L\left[N_{1}\right]=L\left[N_{3}\right]$, $L\left[N_{2}\right]=L\left[N_{4}\right]$. Thus, the radiance of $q_{2}$ can be obtained from the set $\left[N_{1}, N_{2}\right]$.

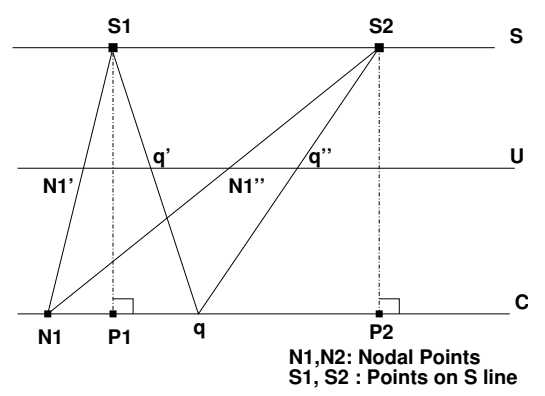

Figure 4. The choice of nodal points is independent of the direction. $N_{1}{ }^{\prime} q^{\prime}$ is equal to $N_{1}{ }^{\prime \prime} q^{\prime \prime}$. 


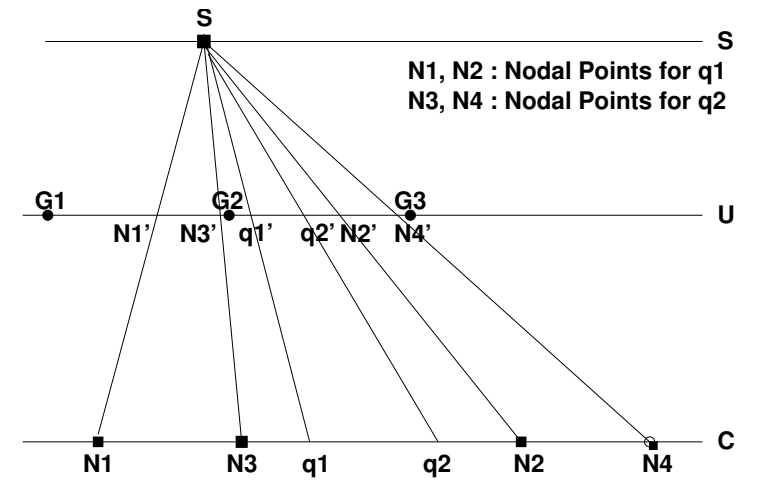

(a) assoc $\left(N_{3}\right)$ is closer to $N_{1}$ than $N_{2}$. Notice that $\operatorname{assoc}\left(\mathrm{N}_{3}\right)=\operatorname{assoc}\left(\mathrm{N}_{1}\right)$.

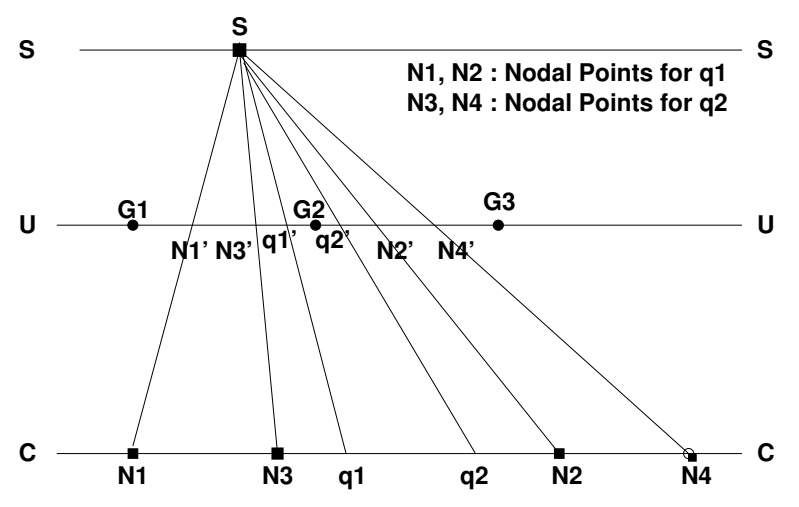

(b) assoc $\left(N_{3}\right)$ is closer to $N_{2}$ than $N_{1}$. Notice that $\operatorname{assoc}\left(\mathrm{N}_{3}\right)=\operatorname{assoc}\left(\mathrm{N}_{2}\right)$.

Figure 6. Power of Nodal Points

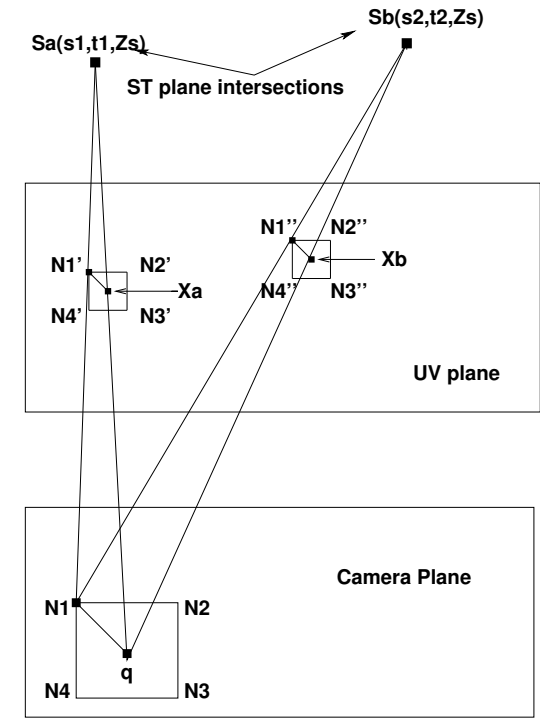

Figure 5. Choice of nodal points is independent of direction

- Case 2: $d\left[N_{2}{ }^{\prime}, \operatorname{assoc}\left(N_{3}\right)\right]<\frac{\Delta l}{2}$ (Shown in Figure $6(b)) \Rightarrow \operatorname{assoc}\left(N_{3}\right)=\operatorname{assoc}\left(N_{2}\right)$. So, $L\left[N_{3}\right]=L\left[N_{2}\right]$. Further, $d\left[q_{2}{ }^{\prime}\right.$, assoc $\left.\left(\mathrm{N}_{1}\right)\right]>\frac{\Delta l}{2}$ and $d\left[q_{2}{ }^{\prime}, \operatorname{assoc}\left(\mathrm{N}_{4}\right)\right]>\frac{\Delta l}{2}$. So in this case, $\operatorname{assoc}\left(\mathrm{q}_{2}\right)=$ assoc $\left(\mathrm{N}_{2}\right)$. Thus, the radiance of $q_{2}$ can be obtained from $N_{2}$.

Thus the lemma asserts that, for $q_{2}$, the radiance values stored at $N_{1}$ and $N_{2}$ are sufficient.
Notice that, unlike in Figure 2, the nodal points $N_{1}$ and $\mathrm{N}_{2}$ are not equidistant from $q_{2}$. Also note that the two cases are not symmetrical.

Lemma 2.3.1 enables us to determine the radiance at any query point $q_{i}$ using the following algorithm:

\begin{tabular}{|l|}
\hline Algorithm 2.3: All-Directions $\left(q_{i}\right)$ \\
\hline \hline 1. Determine nodal points bounding $q_{i}$. \\
2. for all $s \in \mathrm{S}$ do \\
Shoot a ray from query point $q_{i}$ to $s$. \\
Apply Algorithm 2.1 .1 and output radi- \\
ance $L[q]$, in the direction of $s$. \\
3. end for \\
\hline
\end{tabular}

We denote the time taken for this operation as $k_{2}$ (Section 4.2).

\subsubsection{Correctness of Algorithm 2.3}

In either of the cases in Lemma 2.3.1, for any query point $q_{i}$ lying in the region bounded by $\left[N_{k}, N_{k+1}\right]$ (determined in Algorithm 2.3: Step 1),

$$
\operatorname{assoc}\left(\mathrm{q}_{\mathrm{i}}\right)=\left\{\begin{array}{l}
\operatorname{assoc}\left(\mathrm{N}_{\mathrm{k}}\right) \text { or } \\
\operatorname{assoc}\left(\mathrm{N}_{\mathrm{k}+1}\right)
\end{array}\right.
$$

The result follows.

Nodal points on the plane also enable multiple query points. Specifically,

Lemma 2.3.2 The nodal points $N_{1}, N_{2}, N_{3}$ and $N_{4}$ of a query point $q_{1}$ on the camera plane are sufficient for determining radiance at any query point in the rectangular region bounded by these nodal points. 


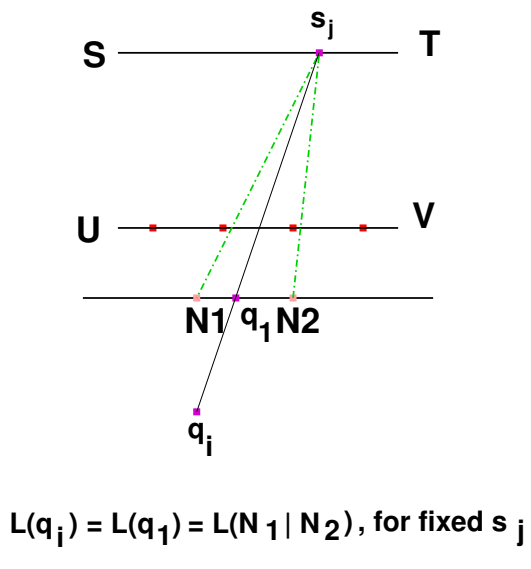

Figure 7.

Proof: The proof of this lemma is similar to that of 2.3.1. Replacing the lines U, S and C by planes UV, ST and Camera, we get four different cases, which asserts that for determining the radiance at any query point the nodal points bounding it are sufficient. For more information, see [7].

Finally, when the query point is placed away from the plane containing nodal points we have,

Lemma 2.3.3 The nodal points $N_{1}, N_{2}$ of a query point $q_{1}$ are sufficient for determining the radiance of any query point $q_{i}$ along a direction $s_{j}$, as long as the points $\left[q_{1}, q_{i}, s_{j}\right]$ are collinear.

Proof: Without loss of generality, let $q_{i}$ be the query point and $s_{j}$ denote a direction. From [1], and for the direction $s_{j}$, the radiance $L\left[q_{1}\right]=L\left[q_{i}\right]$ since $q_{1}$ is visible from $q_{j}$. From Lemma 2.3.1, $L\left[q_{1}\right]$ can be obtained from $N_{1}$ and $N_{2}$. The result follows.

\subsection{Ray Intersection}

Algorithm 2.3 requires costly [9] ray intersections and should be avoided. In this section we show how to avoid ray intersection using the Taylor's theorem. Specifically, consider $X_{1}=\left[X_{c}, Y_{c}, Z_{c}\right]$, which is center of projection for a virtual camera, and $I=\left(I_{x}, I_{y}, C_{1}\right)$, which is a point on the ST plane. Then a ray from $X_{1}$ to $I$, intersects UV plane at $g\left(X_{1}\right)=\left[g_{x}, g_{y}, C_{2}\right]$. Moving the COP to the location $X_{2}=X_{1}+\Delta X$, the change in $\mathrm{x}$ co-ordinate of the point of intersection with the UV plane is given by:

$$
\Delta g_{x}=\frac{C_{2}-C_{1}}{Z_{c}-C_{1}} \Delta X_{c}+\frac{\left(I_{x}-X_{c}\right)\left(C_{1}-C_{2}\right)}{\left(Z_{c}-C_{1}\right)^{2}} \Delta Z_{c}
$$

A similar equation is derived for $\Delta g_{y}$. The error associated with approximation is given by $E_{g_{x}}$,

$$
E_{g_{x}}=g_{x}\left(X_{1}+\Delta X\right)-\bar{g}_{x}\left(X_{1}+\Delta X\right)
$$

where, $\bar{g}_{x}\left(X_{1}+\Delta X\right)$ is the Taylor's estimate. From the first order analysis

$$
E_{g_{x}}=\frac{2\left(I_{x}-X_{c}\right)\left(C_{1}-C_{2}\right)}{\left(C_{1}-Z_{c}\right)^{2}} \Delta Z_{c}
$$

A similar equation is derived for $E_{g_{y}}$. If the camera motion is on any arbitrary path in a plane parallel to the ST plane $\left(\Delta Z_{c}=0\right)$, then the error $E_{g_{x}}=0, E_{g_{y}}=0$. Higher order Taylor error also are zero. In addition, the computational complexity involved in calculating the new UV intersection point decreases substantially, as (4) reduces to

$$
\Delta g_{x}=\frac{C_{2}-C_{1}}{Z_{c}-C_{1}} \Delta X_{c}
$$

which is independent of the direction of the ray. This implies that a regular camera motion results in a regular shift of intersection points. In other words, we avert expensive ray intersection computations.

\section{Caching Nodal Points}

We now have the mathematical apparatus to select the nodal points given a camera walk. For the sake of exposition, we consider two orthogonal cases.

- Case 1: Camera Walk on a plane parallel to the UV plane.

Algorithm 3: 2D-Incremental-CameraWalk
(walk)

1. Starting from the initial position on the camera path curve, mark nodal points at a distance $\Delta x=\Delta l \times R$, where $R$ is the ratio of the distance between the camera plane and the ST plane, and the distance between the UV and ST plane. For simplicity, the nodal points are selected parallel to the $\mathrm{u}$ and $\mathrm{v}$ directions as shown in Figure 8(a). A grid is thus created.

2. The light field is cached at four nodal points in the grid enclosing the query point (The precise computation of the light field at the nodal points can take advantage of the methods suggested in Section 2.4, instead of the original method [5].)

3. Apply Algorithm 2.3 to calculate the radiance at any query point inside the initial cell.

4. As the walk exits a grid cell, update the nodal points and go to Step 2. 


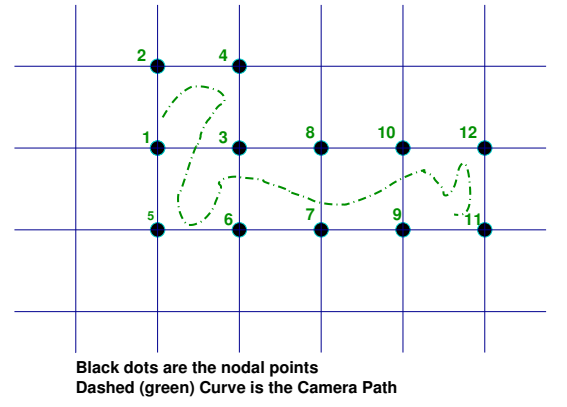

(a) 2D-Camera Walk: Incremental Nodal Points along the camera walk

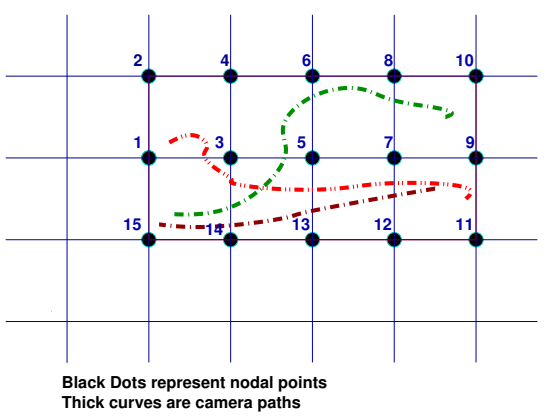

(b) 2D-Camera Walk: Domain-based Nodal Points

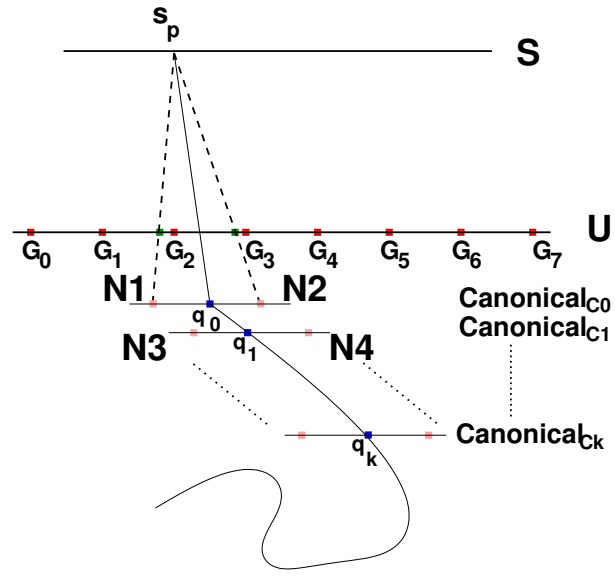

(c) 3D-Camera Walk: Incremental Nodal Points

Figure 8. Beauty of Nodal Points

If we are given several camera walks lying in a domain, an alternate, version of the algorithm is to pick domain-based nodal points, as shown in Figure 8(b). Any query, on any camera walk, or even at random, in the rectangular region defined by the convex hull of the nodal points can be answered efficiently.

- Case 2: Camera Walk along any arbitrary path in 3-dimension.

For brevity, we again consider a setup similar to the two slab setup where planes (UV and ST) are replaced by lines $U$ and $S$ as in Section 2 (Figure 8(c)). We now have an arbitrary camera walk in the $3 \mathrm{D}$ coordinate frame. The incremental algorithm is as follows:

\section{Algorithm: 3D-Incremental-CameraWalk (walk)}

1. Compute nodal points $N_{i}$ and $N_{i+1}$ on the line (termed Canonical $_{c_{i}}$ ) parallel to U using Algorithm 2.1.1.

2. Cache light field at these nodal points as in Step 2 of Algorithm 3.

3. Compute interval $I_{j}$ on Canonical $_{c_{i}}$ defined by the intersections of the two line segments from the two corners of $\mathrm{S}$ to $q_{j}$ on walk. If $I_{j} \in\left[N_{i}, N_{i+1}\right]$, use Algorithm 2.3 to calculate $L\left[q_{j}\right]$ (Lemma 2.3.3). Increment $j$, go to Step 3.

4. Otherwise, increment $i$ and go to Step 1.
The two cases described above paves the way for the algorithm for the camera walk. For variety, we describe a domain based algorithm. 


\subsection{The Algorithm}

\section{Algorithm 3.1: All-CameraWalk}

1. Determine $q_{0}$ the point on the arbitrary camera walk corresponding to the minimum $z$ coordinate (Figure 9).

2. Determine the plane (termed Canonical ${ }_{c}$ ) parallel to the UV plane at $q_{0}$.

3. Determine the convex hull (the dotted-dashed quadrilateral in Figure 9) of the path.

4. Determine tangent lines from the two corners of $\mathrm{S}$ to the convex hull and thus points of intersections with Canonical $_{c}$.

5. Determine nodal points on Canonical ${ }_{\mathrm{c}}$ using Step 1 of Algorithm 3 in the interval defined by the points.

6. For a query point $q_{i}$ on the camera walk,

- For a point $s_{j}$ on $\mathrm{S}$, determine $q_{i}^{\prime}$, the intersection of the ray $\overline{q_{i} s_{j}}$ with Canonical ${ }_{c}$.

- Use Algorithm 2.1.1.

The domain-based algorithm described above is useful when there are a number of camera walks, or random query points in the region bounded by the domain. The incremental algorithm, on the other hand, is more suitable when we want to conserve memory for the nodal points.

\subsection{Quadrilinear Versus Nearest Neighbor Approx- imation}

Once nodal points are known, nearest neighbor approximation or quadrilinear interpolation can be used. In generating views using the nearest neighbor approximation, four nodal points will suffice for all information that is needed for intermediate query points. For quadrilinear interpolation 16 nodal points are needed to provide information (radiance) for a query point.

\section{Sample Results}

In this section, we first provide evidence that the results obtained by the use of our method matches those obtained by the implementation given in [5]. Later we show that our method requires less resources.

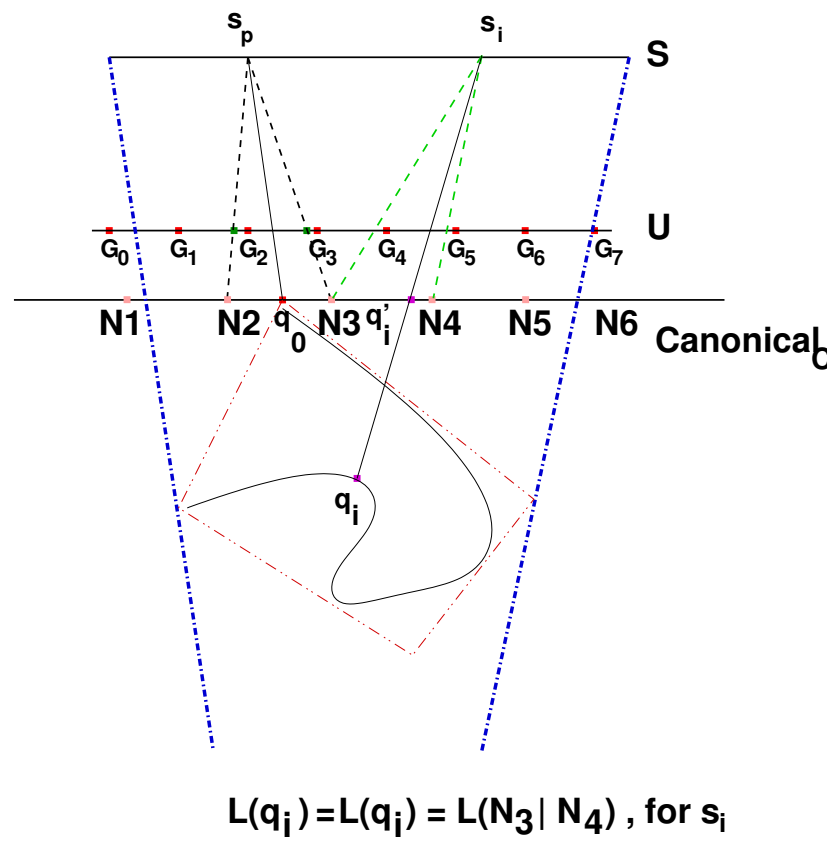

Figure 9. Domain-based Nodal Points

\subsection{Buddha and Dragon}

Figure 11 shows results obtained using our method from Section 3. The images are identical to those generated using [5], as returned by diff in Unix. The virtual camera viewpoint was (for example in Figure $11(\mathrm{~g}))$ at $(0,0,3)$ and the nodal points were situated at $( \pm 0.09375, \pm 0.09375,3.00000)$, where the origin was at the center of the ST plane. The input images were those obtained using $32 \times 32$ cameras.

Identical behavior is observed when we render the dragon (Figure 12); the light field for this was acquired using $8 \times 8$ cameras. Here the virtual camera was (for example in Figure 12(g)) located at $(0.03,0.02,2.00)$ and the nodal points at $( \pm 0.5, \pm 0.5,2)$.

\subsection{Computational Advantage}

We now proceed to show the computational advantage when a camera walk is introduced. As discussed earlier, advantages arise due to nodal light field caching, and avoiding ray intersection calculations. Let $n$ be the number of query points, and $p$ the number of nodal points. Denote $k_{1}$ to be the time taken for ray intersection computations in the original method [5] for one query; if we use homography (from [9]), then this value is negligible.

When the input light field is densely sampled, and is at a high resolution, it may or may not be possible to place the light field in memory. We penalize access to the light field 


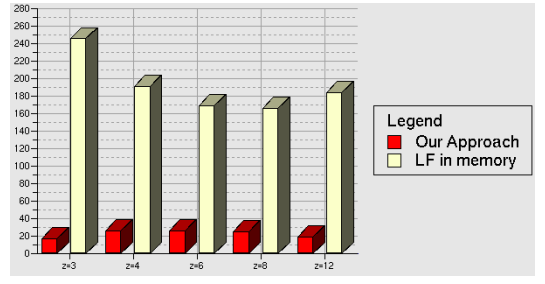

(a) Experiments performed with $32 \mathrm{MB}$ RAM. The time taken by the proposed method is considerably lesser than the original method.

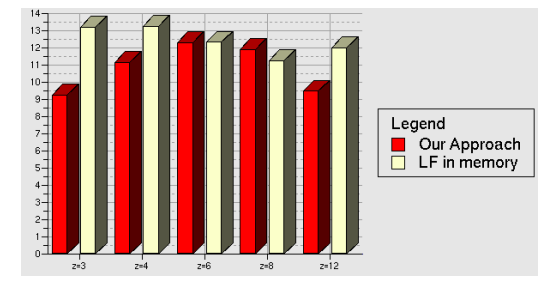

(b) Experiments performed with 1GB RAM. Time taken by our approach is comparable. However, our approach uses only a very tiny fraction $(2 \%)$ of the system memory.

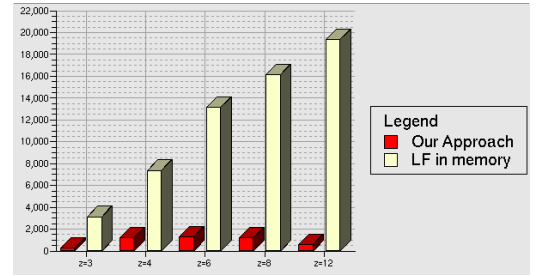

(c) Number of disk accesses. Our approach performs significantly better.

Figure 10. All results are for 100 query points.

(for both methods) by the factor $t$ in the following equation. In Algorithm 3: 2D-Incremental-CameraWalk, the expected gain is

$$
\frac{n\left(t+k_{1}\right)}{\left(p\left(t+k_{1}\right)+n k_{2}\right)}
$$

If the light field does not fit in memory, $t$ represents disk access time. Therefore $t \gg k_{1}$, and the gain is approximately $\frac{n}{p}$.

\subsubsection{Resource Usage}

For the purpose of comparison, and to hand an advantage to the original light field implementation, we have chosen not to use the optimization in Section 2.4 in the experiments. Nevertheless, the results are worth noting. Our time results are based on an Intel Pentium IV 2.4GHz Linux based computer with 1 GB memory.

1. To simulate low RAM situations, we used only $32 \mathrm{MB}$ of the $1 \mathrm{~GB}$ available and rendered Buddha on various camera paths located at different distances from the original camera gantry.

We note that there is considerable gain as seen in figure $10(a)$, where the real time taken by the two approaches is plotted with respect to the $\mathrm{z}$ co-ordinate of COP. The average value of $p$ for $n=100$ is approximately 19 , so the time gain is approximately 5.26 , which is what theoretically equation 8 promises.

2. When the memory is sufficiently large to accommodate the huge light field, Figure 10(b) shows that the time taken by our method is comparable to the original method. This indicates that computing and going to the nodal cache is not very expensive. However, the total memory that we used was even less than $2 \%$ of the memory requirement of method in [5]. This is due to the fact that the method in [5] uses $u \times v \times r$ units of memory for a $u \times v$ camera gantry with an image resolution of $r$.

3. To quantify disk access, we rendered Buddha on various camera paths located at different distances from the original camera gantry. Starting at $(-1.5,-1.5, z$ Cord $)$ and going to $(1.5,1.5, z$ Cord $)$, the virtual camera was made to follow different zigzag paths at different values of $\mathrm{z}$ co-ordinate denoted $z C o r d$. The query points were chosen randomly along these paths. In the experiments on the Buddha image, the origin of the co-ordinate system was located at the center of the ST plane. Figure 10(c) shows the relative gain in terms of disk accesses. The graph shows the number of accesses to the disk storage required by various techniques when the nearest neighbor approximation is used, at $z=3,4,6,8,12$, where $z$ is the distance of the COP from the ST plane.

As a point to note, when the value of $z$ increases, the number of nodal points required for the same camera path decreases and so we get a quantitative difference in the number of disk accesses.

\section{Final remarks}

In virtual reality and in gaming applications, the light field is useful because no information about the geometry or surface properties is needed. However, there are some disadvantages.

In this paper, we have looked at the problem of reducing the computational burden in dealing with the rich and densely sampled light field when a user walks through a virtual world. We have achieved this by recognizing that instead of considering the complete light field, it is enough to consider a sparse set of nodal points. The number of nodal 
points, and the distance between them have been characterized to ensure that the rendering of the scene is identical to what may have been done without the cache. The proofs of these characterizations have also been given.

Our description does not explicitly deal with decompression issues (indeed, in the first stage [5] of rendering, the entire light field is decompressed as it is read into memory from disk.) However, there is not any conceptual blockade in applying the general caching strategy and the mathematical elements even in this case.

\section{References}

[1] E. H. Adelson and J. R. Bergen. Computational Modeling of Vision Processing, chapter The Plenoptic Function and the Elements of Early Vision. MIT Press, 1991.

[2] D. Burschka, G. D. Hager, Z. Dodds, M. Jgersand, D. Cobzas, and K. Yerex. Recent methods for imagebased modeling and rendering. In Proceedings of the IEEE Virtual Reality 2003, page 299. IEEE Computer Society, 2003.

[3] P. Debevec and S. Gortler. Image-based modeling and rendering. In In SIGGRAPH 98 Course Notes. ACM SIGGRAPH, Addison Wesley, July, 1998.

[4] S. J. Gortler, R. Grzeszczuk, R. Szeliski, and M. F. Cohen. The Lumigraph. Computer Graphics, 30(Annual Conference Series):43-54, 1996.

[5] M. Levoy and P. Hanrahan. Light field rendering. In Proceedings of the 23rd annual conference on Computer graphics and interactive techniques, pages 3142. ACM Press, 1996.

[6] P. J. Narayanan. Image based rendering: A critical look. In Second Indian Conference on Computer Vision, Graphics and Image Processing ICVGIP, pages 216-222, 1998.

[7] A. Pandey, B. Choudhury, and S. Chandran. Light Field Based CameraWalk. http://www.cse.iitb. ac. in/ biswarup/research/icvgip2004/.

[8] A. Pandey, B. Choudhury, and S. Chandran. Efficient light field based camerawalk. In Fourth Indian Conference on Computer Vision, Graphics and Image Processing ICVGIP, pages 302-307, 2004.

[9] P. Sharma, A. Parashar, S. Banerjee, and P. Kalra. An uncalibrated lightfield acquisition system. In Third Indian Conference on Computer Vision, Graphics and Image Processing ICVGIP, pages 25-30, 2002. 


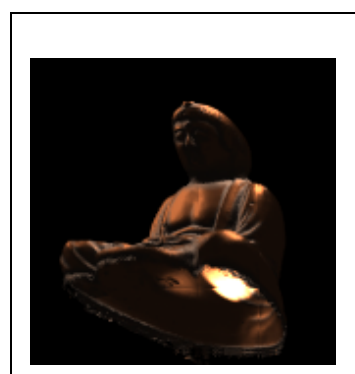

(a)

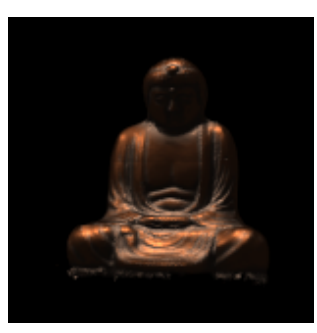

(f)

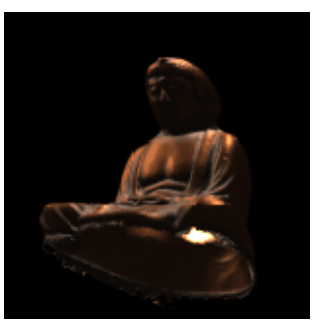

(b)

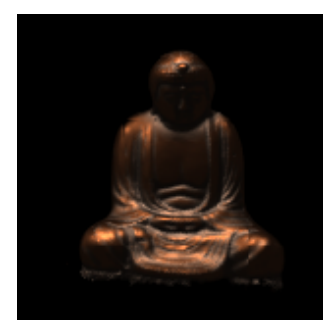

(g)

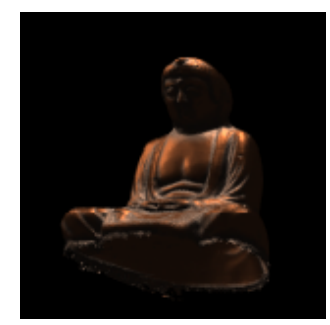

(c)

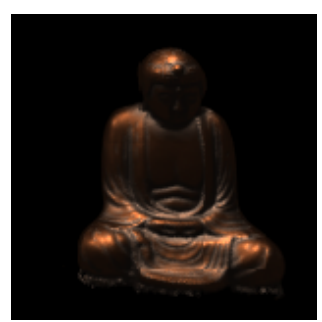

(h)

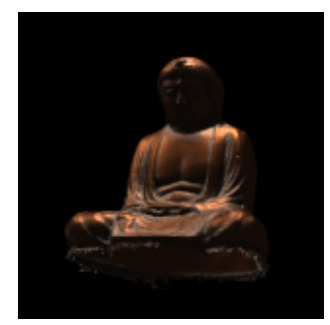

(d)

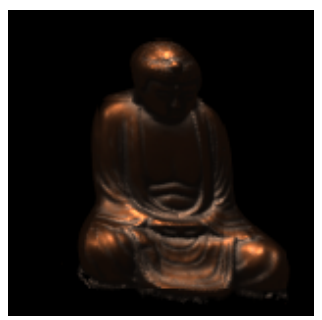

(i)

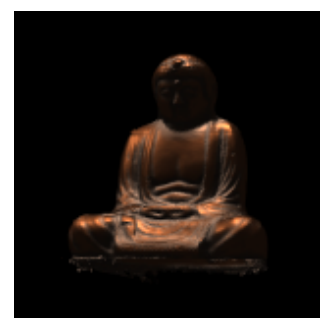

(e)

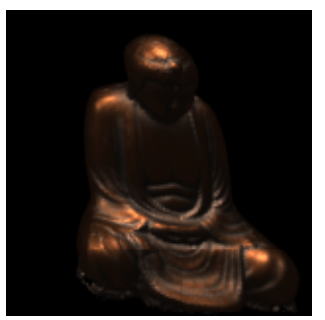

(j)

Figure 11. Rendered images of Buddha on a camera walk using our method

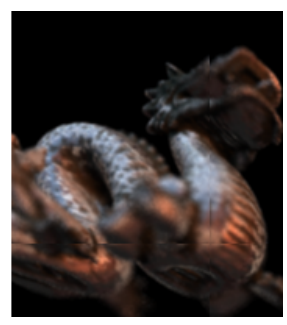

(a)

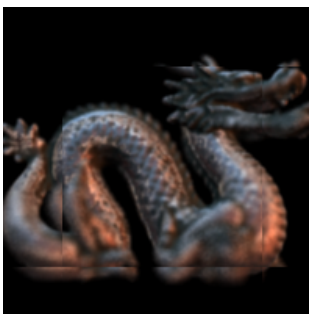

(f)

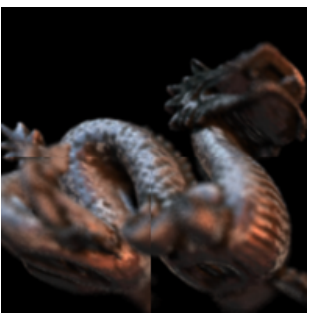

(b)

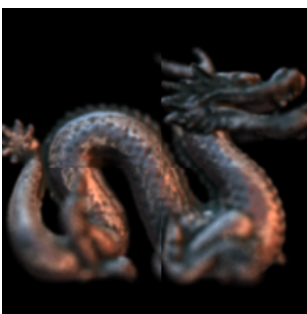

(g)

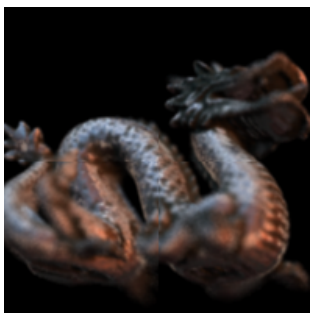

(c)

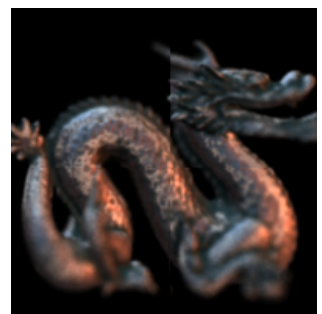

(h)

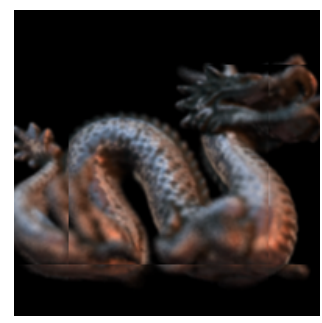

(d)

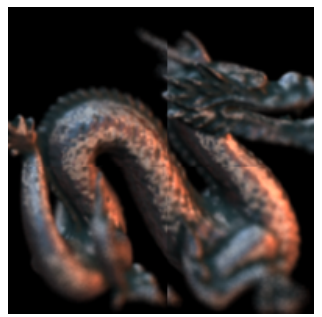

(i)

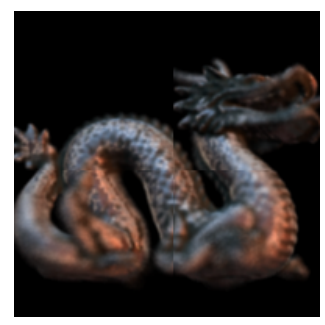

(e)

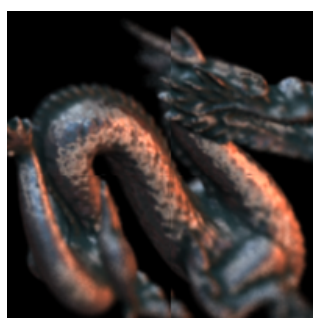

(j)

Figure 12. Rendered images of Dragon on a camera walk using our method 